\title{
New Micromechanics Design Theory for Pseudostrain Hardening Cementitious Composite
}

\author{
By Tetsushi Kanda ${ }^{1}$ and Victor C. Li, ${ }^{2}$ Member, ASCE
}

\begin{abstract}
The micromechanics design theory has realized random short fiber-reinforced cement composites showing pseudostrain hardening (PSH) behavior with over 5\% of strain capacity under tension. Nevertheless, this existing theory currently is limited to specific constituent properties, which does not account for chemical bond and fiber rupture. This article presents a new design theory that eliminates this restriction, achieving fiber rupture type PSH-random short fiber-reinforced cement composites with high-performance hydrophilic fibers like polyvinyl alcohol fibers. Uniaxial tensile tests are conducted employing polyvinyl alcohol fiber composites, the results of which support the validity of the proposed theory. Furthermore, parametric study employing the proposed theory quantitatively evaluates the effects of composite's micromechanics parameters, such as bond strength and fiber strength, on composite performance. This parametric study reveals that continuously increasing the degree of fiber rupture (fiber rupture intensity) enhances the strength performance of composites but not energy performance. However, an optimum rupture intensity exists for maximizing energy performance, which is critical for PSH behavior. The consistency between theoretical predictions and experimental results consequently demonstrates that the proposed theory can be utilized practically as a powerful and comprehensive tool for PSH composite design.
\end{abstract}

\section{INTRODUCTION}

Significantly ductile fiber-reinforced composites have been realized with brittle matrix such as ceramics and cement after Aveston et al. (1971). The mechanics of this ductile behavior, which is called pseudostrain hardening (PSH), has been investigated substantially especially in the field of fiber-reinforced ceramics [e.g., Marshall et al. (1985), Spearing and Zok (1993), Evans et al. (1994)]. These research studies are restricted in unidirectionally aligned fiber composites, but the extension of these studies for random short fiber composites has not been accomplished fully.

This extension has been initiated recently and ductile random short fiber-reinforced cementitious composites (RSFRCC) with PSH behavior have been developed, which show very high fracture toughness and tensile strain capacity as summarized by Naaman and Reinhardt (1995). For example, PSHRSFRCCs with polyethylene (PE) fiber can achieve $30 \mathrm{~kJ} / \mathrm{m}^{2}$ fracture toughness and 5\% tensile strain capacity with less than $2 \%$ volume fraction of fibers, far outperforming conventional materials of similar composition (Li 1993). This high performance has been realized via "composite design," which is based on micromechanics and has been investigated in detail (Li and Leung 1992; Li 1993). This design method emphasizes the proper combination of micromechanical parameters of constituent materials such as fiber, matrix, and fiber-matrix interface to achieve the unique composite properties.

However, the usefulness of the existing PSH composite design theory is limited to specific constituent characteristics, including friction-dominated interface and complete fiber pullout. Although having significant potential for PSH composites, some high-performance hydrophilic fibers cannot be utilized because of this restriction. For example, a polyvinyl alcohol (PVA) fiber appears promising while its potential was not utilized fully (Akihama et al. 1985; Betterman et al. 1995). Nevertheless PVA fiber's potential has been emphasized; e.g., a

${ }^{1}$ Kajima Tech. Res. Inst., 2-19-1 Tobitakyu, Chofu-shi, Tokyo 1820036, Japan.

${ }^{2}$ Prof. and Dir., ACE-MRL, Dept. of Civ. and Envir. Engrg., Univ. of Michigan, Ann Arbor, MI 48109-2125.

Note. Associate Editor: Gilles Pijaudier-Gabot. Discussion open until September 1, 1999. To extend the closing date one month, a written request must be filed with the ASCE Manager of Journals. The manuscript for this paper was submitted for review and possible publication on January 26, 1998. This paper is part of the Journal of Engineering Mechanics, Vol. 125, No. 4, April, 1999. CASCE, ISSN 0733-9399/99/ 0004-0373-0381/\$8.00 + \$.50 per page. Paper No. 17439.
PVA fiber with $14 \mu \mathrm{m}$ in diameter and 1,500 $\mathrm{MPa}$ of tensile strength was reported to indicate high potential for PSH behavior in bending (Garcia et al. 1997). This fiber is expected to involve severe fiber rupture in composites because of small fiber diameter and high fiber-matrix interfacial properties dominated by chemical bond (Kanda and Li 1998b). Quantitative micromechanics evaluation for this composite and the fundamental basis for the observed high composite performance so far have not been clarified in the published literature. Likewise, no design guideline exists for this type of composites at the present. The establishment of a new composite design theory accounting for chemical bond and fiber rupture is a major task for PSH composite research.

This task can be achieved by adopting an energy approach for PSH composite design ( $\mathrm{Li} \mathrm{1993)} \mathrm{that} \mathrm{incorporates} \mathrm{a} \mathrm{new}$ bridging law, the relationship between crack opening displacement (COD) and the composite's crack bridging stress caused by fibers. Such a bridging law accounting for fiber rupture and chemical bond recently has been presented by the authors (Kanda and $\mathrm{Li}$ 1999) and demonstrated to reproduce reasonably the tensile characteristics of high-performance hydrophilic fiber composites like PVA fiber systems. The energy approach expresses the fundamental condition for occurring steady-state cracking and multiple cracking, which are mechanical sources of PSH behavior.

The focus in the current study is in establishing a new design theory for high-performance hydrophilic fiber composites with PSH behavior and in achieving a better understanding of these composites' tensile response. The derivation of this theory is first explained via theoretical conditions to be satisfied to achieve PSH behavior. Next, an experimental program with PVA fiber composites is presented. These test results are used for theory validation. Parametric studies with this design theory then are conducted to understand the effects on composite performance of micromechanics parameters such as fiber strength and bond strength. Finally, the PVA fiber composites tested are examined in light of this theory for PSH behavior optimization by tailoring micromechanics parameters.

\section{PSEUDOSTRAIN HARDENING COMPOSITE DESIGN THEORY}

\section{Bridging Stress-COD Relation for Composites}

A bridging law, briefly summarized in this section, recently has been developed for RSFRCCs involving fiber rupture and chemical interface properties (Kanda and Li 1998a). This 
bridging law describes the relationship between crack bridging stress caused by fiber $\sigma_{c}$ and COD $\delta$ and is expressed in normalized form

$$
\overline{\boldsymbol{\sigma}}_{c}=\text { func. }\left(\frac{E_{f}}{\lambda}, \frac{\sigma_{d s}}{\lambda}, \frac{\sigma_{f u}^{n}}{\lambda}, f, f^{\prime}, \bar{\delta}\right)
$$

where

$$
\begin{gathered}
\bar{\sigma}_{c}=\frac{\sigma_{c}}{V_{f} E_{f} / 4}, \quad \bar{\delta}=(1+\eta) \hat{\delta} \\
\hat{\delta}=\frac{2 \delta}{L_{f}}, \quad \eta=\frac{V_{f} E_{f}}{\left(1-V_{f}\right) E_{m}}
\end{gathered}
$$

where $V_{f}=$ volume fraction of fiber; $E_{f}=$ elastic modulus of fiber; and $E_{m}=$ elastic modulus of matrix. The full expression of (1) is presented in Appendix I. This bridging law $\bar{\sigma}_{c}-\bar{\delta}$ relation is expressed in terms of five nondimensional primary parameters: $E_{f} / \lambda, \sigma_{d s} / \lambda, \sigma_{f u}^{n} / \lambda, f$, and $f^{\prime}$. The $\lambda$ is related to interfacial friction and fiber aspect ratio.

$$
\lambda \equiv 2 \tau_{i}\left(\frac{L_{f}}{d_{f}}\right)
$$

where $\tau_{i}=$ interfacial frictional bond strength; $L_{f}=$ fiber length; and $d_{f}=$ fiber diameter. Higher $\lambda$ means high interfacial friction and/or fiber aspect ratio. The first primary parameter in (1), $E_{f} / \lambda$ where $E_{f}$ denotes the fiber's elastic modulus mainly affects the ascending stiffness of the $\bar{\sigma}_{c}-\bar{\delta}$ curve. Higher $E_{f}$ $/ \lambda$ leads to smaller $\delta_{\text {peak }}$, the COD at peak bridging stress $\sigma_{\text {peak }}$. The second parameter $\sigma_{d s} / \lambda$ is a measure of the ratio between chemical bond strength to interfacial friction. The $\sigma_{d s}$ is defined as

$$
\sigma_{d s} \equiv 2(1+\eta)\left(\frac{\tau_{s}}{\rho}\right)
$$

where

$$
\begin{gathered}
\rho^{2}=\frac{2 G_{c} E_{c}}{\left[\left(1-V_{f}\right) E_{m} E_{f} \log \left(\frac{2 R^{*}}{d_{f}}\right)\right]} \\
\log \left(\frac{2 R^{*}}{d_{f}}\right)=-\left[2 \log V_{f}+\left(1-V_{f}\right)\left(3-V_{f}\right)\right] /\left\{4\left(1-V_{f}\right)^{2}\right\}
\end{gathered}
$$

where $\tau_{s}=$ chemical bond strength; $E_{c}=$ elastic modulus of composite; and $G_{c}=$ shear modulus of composite. The third parameter $\sigma_{f u}^{n} / \lambda$ expresses the ratio of fiber strength to frictional bond strength. Higher $\sigma_{f u}^{n} / \lambda$ means more fiber pullout without rupture in the composites. The fourth parameter $f$ is the so-called snubbing coefficient (Li et al. 1990), which represents friction-enhancing effects caused by the inclining angle of the fiber lying obliquely to a crack plane. The last parameter $f^{\prime}$ is the fiber strength reduction factor and represents the negative effects of the inclining angle in which tensile load resistance of the fiber apparently deteriorates with increasing angle (Kanda and Li 1998a).

\section{MATERIAL DESIGN CONDITION FOR STRAIN HARDENING}

The material design conditions required for PSH are summarized by Li (1993). By adopting the $J$-integral approach, the applied stress $\sigma_{a}$ and the crack tip toughness $J_{\text {tip }}$ during steady-state cracking were derived (Marshall and Cox 1988)

$$
J_{\text {tip }}=\sigma_{a} \delta_{a}-\int_{0}^{\delta_{a}} \sigma_{c}(\delta) d \delta
$$

where $\sigma_{a}=$ steady-state cracking stress; $\delta_{a}=$ COD correspond-

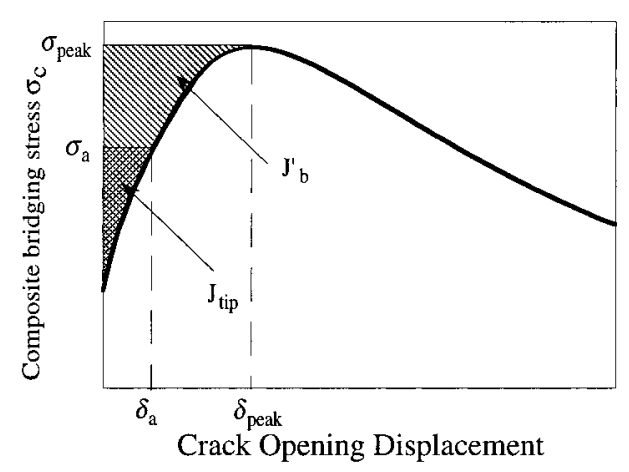

FIG. 1. Condition for Strain Hardening

ing to $\sigma_{a}$ in $\sigma_{c}-\delta$ curve; and $J_{\text {tip }}=$ crack tip toughness. For multiple cracking to occur

$$
\begin{gathered}
\sigma_{a} \leq \sigma_{\text {peak }} \\
J_{\text {tip }} \leq J_{b}^{\prime}=\sigma_{\text {peak }} \delta_{\text {peak }}-\int_{0}^{\delta_{\text {peak }}} \sigma_{c}(\delta) d \delta
\end{gathered}
$$

where $\sigma_{\text {peak }}=$ peak stress of $\sigma_{c}(\delta) ; \delta_{\text {peak }}=$ COD corresponding to $\sigma_{\text {peak }}$; and $J_{b}^{\prime}=$ complementary energy of $\sigma_{c}-\delta$ curve. The complementary energy $J_{b}^{\prime}$ expresses the net available energy for extending the steady-state matrix crack as part of the externally supplied total energy consumed in the inelastic crack bridging "spring", elements. Following the notation in (1), the $\mathrm{PSH}$ condition of (6) can be expressed in normalized form.

$$
\bar{J}_{b}^{\prime}=\bar{\sigma}_{\text {peak }} \bar{\delta}_{\text {peak }}-\int_{0}^{\bar{\delta}_{\text {peak }}} \bar{\sigma}_{c}(\bar{\delta}) d \bar{\delta}
$$

where $\bar{J}_{b}^{\prime}=\left[8 J_{b}^{\prime}(1+\eta)\right] /\left(V_{f} E_{f} L_{f}\right), \bar{\sigma}_{\text {peak }}=4 \sigma_{\text {peak }} /\left(V_{f} E_{f}\right)$, and $\bar{\delta}_{\text {peak }}=(1+\eta) \hat{\delta}_{\text {peak }}$. Implicit in (5) is that the first crack strength (which depends on matrix toughness and initial flaw size) is lower than the maximum bridging stress $\sigma_{\text {peak }}$. The foregoing criteria are illustrated schematically in Fig. 1, with crack tip toughness $J_{\text {tip }}$ less than $J_{b}^{\prime}$, thus satisfying (5) and (6). These criteria can be applied directly to the hydrophilic fiber composites discussed in this study. However, $\bar{\sigma}_{\text {peak }}$ and $\bar{\delta}_{\text {peak }}$ cannot be expressed in simple analytic form when $\bar{\sigma}_{c}$ is represented by (1). Furthermore, a simple expression for the critical fiber volume fraction $V_{f}^{\text {crit }}$, below which strain hardening does not occur in composites, cannot be derived from (7). Therefore, the strain hardening criteria must be expressed in numerical form for high-performance hydrophilic fiber composites.

\section{THEORY VALIDATION}

\section{Experimental Program and Theory Validation Scheme}

The presented PSH-RSFRCC design theory is verified employing uniaxial tensile test results of several composites, which show a broad range of strain extension in PSH behavior. Theory validation is to be achieved by comparing strain extension between theoretical predictions and experimental observations. For this purpose, two types of PVA fiber composites are tested, which are designed to show PSH behavior but with different extent. Because these composites are expected to involve fiber rupture and fiber-matrix interface property dominated by chemical bond, the present theory should be appropriate to design these PVA fiber composites. This design process is described in the next subsection in detail. These PVA composites have identical matrix mix proportion, which is presented in Table 1. The first PVA fiber composite involves a $40-\mu \mathrm{m}$-diameter PVA fiber with volume fraction of $2 \%$. The 
TABLE 1. Matrix Mix Proportion

\begin{tabular}{c|c|c|c|c|c}
\hline \hline $\begin{array}{c}\text { Total } \\
\text { cement } \\
\text { material }^{\mathrm{a}}\end{array}$ & $\begin{array}{c}\text { Total } \\
\text { water } \\
(1)\end{array}$ & $\begin{array}{c}\text { Cement } \\
(3)\end{array}$ & $\begin{array}{c}\text { Silica } \\
\text { fume }^{\mathrm{b}} \\
(4)\end{array}$ & $\begin{array}{c}\text { Water } \\
(5)\end{array}$ & $\begin{array}{c}\text { Super } \\
\text { plasticizer } \\
(6)\end{array}$ \\
\hline 1.00 & 0.27 & 0.9 & 0.2 & 0.14 & 0.040 \\
\hline
\end{tabular}

${ }^{\mathrm{a}}$ Including cement and solid silica fume.

${ }^{\mathrm{b}}$ Including $50 \%$ of water.

Including $66 \%$ of water.

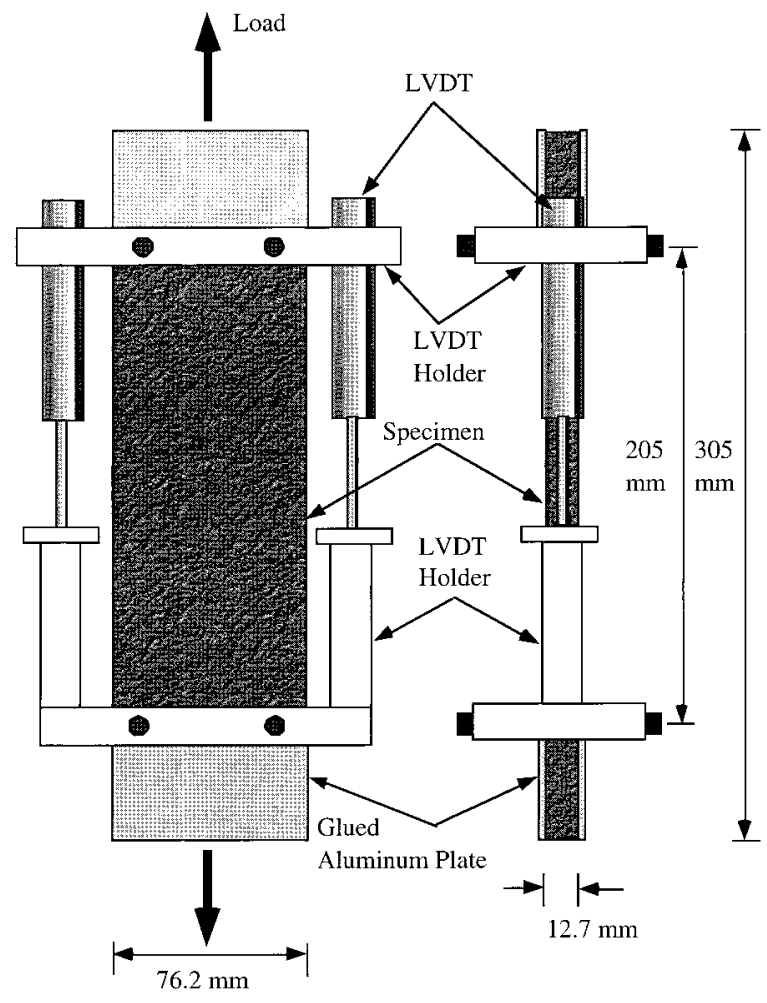

FIG. 2. Tensile Test Setup

second involves a 14- $\mu$ m-diameter PVA fiber with volume fraction of $1.5 \%$. Uniaxial tensile tests are conducted with these PVA composites by using testing setup shown in Fig. 2. These tests adopt coupon specimen $12.7 \times 76.2 \times 305 \mathrm{~mm}$ as depicted in Fig. 2. Tensile strain is measured using two linear variable differential transducers with $205 \mathrm{~mm}$ of gauge length. Other testing details can be found in Li et al. (1995).

Data for a fiber composite reported elsewhere ( $\mathrm{Li}$ et al. 1995) also are included in the current study, for the purpose of comparisons. This composite involves $2 \%$ volume fraction of high-performance PE fiber and exhibited saturated PSH behavior with remarkably high tensile strain capacity. Postmortem analysis of fracture surface indicates no fiber rupture in this composite. The single fiber test also indicates no chemical bond for PE fibers in cementitious composites (Li et al. 1996). This type of composite has been investigated in detail both theoretically and experimentally, and its tensile behavior has been demonstrated to be in reasonable accord with micromechanics-based predictions ( $\mathrm{Li}$ et al. 1995). The fiber pullout model used for the PE composite forms the basis of the current extended theory. The fiber pullout model is recovered if no fiber rupture and friction dominant fiber/matrix interface is assumed in the presented model. These limiting conditions can be expressed with $\sigma_{d s} / \lambda=f^{\prime}=0$ and $1<\hat{L}_{r}$. The $\hat{L}_{r}$ represents the critical embedment length of obliquely aligned fiber in matrix, below which no fiber rupture is expected. The expression of $\hat{L}_{r}$ is presented in Appendix I.

\section{Experimental Results}

Stress-strain curves obtained from uniaxial tensile tests are shown in Figs. 3-5 for the two PVA and the PE composites. It is clear that very different degrees of PSH (PSH intensity, hereafter) were achieved. The PSH intensity may be represented by the tensile strain capacity $\varepsilon_{c u}$, the strain magnitude when continuous stress drop occurs. Fig. 3 shows the moderate PSH intensity with approximately $1 \%$ of $\varepsilon_{c u}$ for the $40-\mu \mathrm{m}$ PVA fiber composite. The weak PSH intensity with lower $\varepsilon_{c u}$ (approximately $0.3 \%$ ) for the $14-\mu \mathrm{m}$-PVA fiber composite is shown in Fig. 4. In contrast, the PE fiber composite (Fig. 5) shows much more intense PSH with $\varepsilon_{c u}$ over $5 \%$ (Li et al. 1995).

This difference in $\varepsilon_{c u}$ is attributed to the number of multiple cracks developed perpendicular to the loading axis. The 40$\mu \mathrm{m}$-PVA fiber composite reveals moderate saturation of multiple cracks. Less than 10 visible cracks were observed over a specimen span length of $205 \mathrm{~mm}$. The 14- $\mu \mathrm{m}$-PVA fiber composite showed just three or four visible cracks over a specimen span length. In contrast, the PE fiber composite tends to generate numerous fine cracks developed with small crack spacing (approximately $2.2 \mathrm{~mm}$ ) over the full specimen length (Wu and $\mathrm{Li}$ 1995). It should be noted that postmortem analysis of

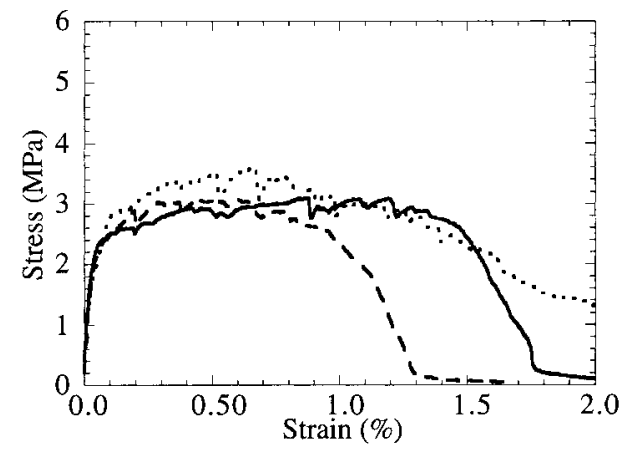

FIG. 3. Tensile Test Result for 40- $\mu \mathrm{m}$-PVA Composite

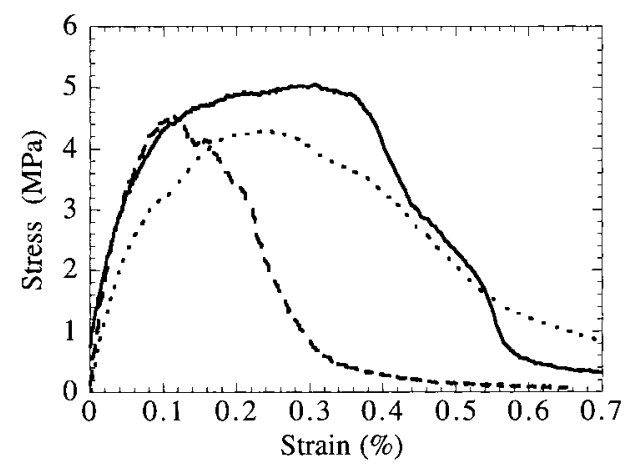

FIG. 4. Tensile Test Result for 14- $\mu$ m-PVA Composite

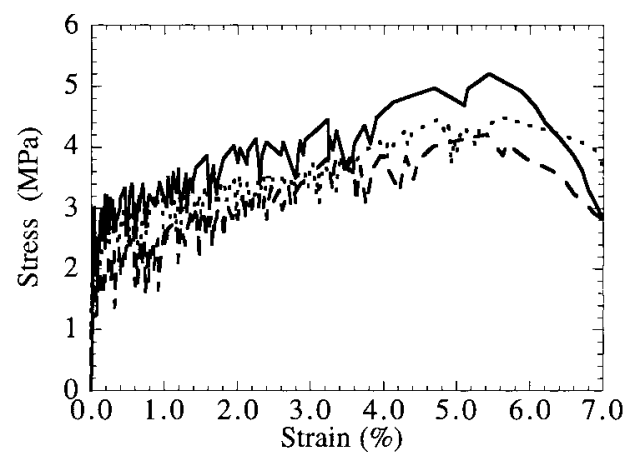

FIG. 5. Tensile Test Result for PE Composite 
generated cracks revealed that similar composite with the 40$\mu \mathrm{m}$-PVA fiber system showed many cracks less than $10 \mu \mathrm{m}$ (Kanda and Li 1998c), which is difficult to distinguish by the naked eyes. Therefore, more cracks are likely developed for the $40-\mu \mathrm{m}$-PVA fiber composites than visible ones.

\section{Analytical Investigation}

These experimental results were examined analytically to verify the proposed theory. First, micromechanics parameters for the three composites are summarized in Table 2 (Kanda and Li 1999). Data in this table have been obtained independent of the composite test data described earlier. This table illustrates the contrasting magnitudes in these parameters among the three composites (Kanda and Li 1999). For example, the 40- $\mu \mathrm{m}$-PVA fiber system has very high frictional/ chemical bond strengths $\left(\tau_{i} \approx 2 \mathrm{MPa}\right.$ and $\tau_{s} \approx 30 \mathrm{MPa}$, respectively) but low aspect ratio $L_{f} / d_{f}(\approx 300)$ and fiber strength $\sigma_{n}^{f u}(\approx 800 \mathrm{MPa})$. The $14-\mu \mathrm{m}$-PVA fiber system has very high frictional/chemical bond strengths $\left(\tau_{i} \approx 4 \mathrm{MPa}\right.$ and $\tau_{s} \approx 30$ $\mathrm{MPa})$ and high fiber aspect ratio $L_{f} / d_{f}(\approx 430)$, but moderate fiber strength $\sigma_{n}^{f u}(\approx 1,650 \mathrm{MPa})$. The PE fiber has very high $\sigma_{n}^{f u}(\approx 2,400 \mathrm{MPa})$ but low bond strengths $\left(\tau_{i} \approx 0.5 \mathrm{MPa}\right.$ and $\left.\tau_{s} \leq 0.5 \mathrm{MPa}\right)$ and $L_{f} / d_{f}(\approx 330)$.

Next, $\sigma_{c}-\delta$ relations of the three composites were computed using (1), employing the parametric values in Table 2. The resulting crack bridging relations are shown in Fig. 6. The 14$\mu \mathrm{m}$-PVA fiber composite has the highest $\sigma_{\text {peak }}$, nearly twice that of the 40- $\mu$ m-PVA composite, but has the lowest $\delta_{\text {peak }}$, approximately $1 / 5$ of that of the PE composite. This difference in $\sigma_{c}-\delta$ relation, in fact, significantly affects the composites' mechanical potential for PSH behavior as discussed later. Furthermore, the estimated $\sigma_{\text {peak }}$ is compared with test result $\sigma_{\text {peak }}^{\text {test }}$ in Fig. 7 , which indicates that $\sigma_{\text {peak }}$ is reasonably consistent with $\sigma_{\text {peak }}^{\text {test }}$. This agreement lends support to the validity of the present theory.

Because $J_{b}^{\prime} / J_{\text {tip }} \geq 1$ ensures satisfaction of the PSH condition in (6), the energy margin $J_{b}^{\prime} / J_{\text {tip }}$ is expected to correlate with the PSH intensity (Kanda and Li 1998b). The $J_{b}^{\prime} / J_{\text {tip }}$ was calculated by employing (6), and this calculation employed $\sigma_{c^{-}}$ $\delta$ relations in Fig. 6 in conjunction with $J_{\text {tip }}$ value, which was

\section{TABLE 2. Constitutive Parameters for Composite}

\begin{tabular}{|c|c|c|c|c|}
\hline $\begin{array}{c}\text { Constituent } \\
\text { (1) }\end{array}$ & $\begin{array}{c}\text { Micromechanical } \\
\text { parameter } \\
(2)\end{array}$ & $\begin{array}{c}40-\mu m- \\
\text { PVA } \\
\text { com- } \\
\text { posite } \\
(3)\end{array}$ & $\begin{array}{l}\text { 14- } \mu \mathrm{m}- \\
\text { PVA } \\
\text { com- } \\
\text { posite } \\
(4) \\
\end{array}$ & $\begin{array}{l}\text { PE } \\
\text { com- } \\
\text { posite } \\
(5)\end{array}$ \\
\hline \multirow[t]{4}{*}{ Fiber } & $\begin{array}{l}\text { Fiber length } L_{f}(\mathrm{~mm}) \\
\text { Fiber diameter } d_{f} \\
\quad(\mathrm{~mm})\end{array}$ & $\begin{array}{c}12 \\
0.040\end{array}$ & $\begin{array}{c}6 \\
0.014\end{array}$ & $\begin{array}{l}12.7 \\
0.038\end{array}$ \\
\hline & $\begin{array}{l}\text { Fiber elastic modulus } \\
\qquad E_{f}(\mathrm{GPa})\end{array}$ & 21.8 & 60 & 117 \\
\hline & $\begin{array}{l}\text { Nominal fiber } \\
\text { strength } \sigma_{f u}^{n}(\mathrm{MPa})\end{array}$ & 806 & 1,666 & 2,400 \\
\hline & $\begin{array}{l}\text { Fiber volume fraction } \\
\quad V_{f}(\%)\end{array}$ & 2 & 1.5 & 2 \\
\hline \multirow[t]{2}{*}{ Matrix } & $\begin{array}{l}\text { Matrix elastic modu- } \\
\quad \text { lus } E_{m}(\mathrm{GPa})\end{array}$ & 23 & 23 & 23 \\
\hline & $\begin{array}{l}\text { Matrix fracture } \\
\text { toughness } K_{m}(\mathrm{MPa} \\
\left.\mathrm{m}^{0.5}\right)\end{array}$ & 0.33 & 0.33 & 0.33 \\
\hline \multirow[t]{4}{*}{$\begin{array}{l}\text { Fiber-matrix } \\
\text { interface }\end{array}$} & $\begin{array}{l}\text { Friction bond strength } \\
\tau_{i}(\mathrm{MPa})\end{array}$ & 2.21 & 4.35 & 0.7 \\
\hline & $\begin{array}{l}\text { Chemical bond } \\
\text { strength } \tau_{s}(\mathrm{MPa})\end{array}$ & 31.3 & 33.6 & - \\
\hline & Snubbing coefficient $f$ & 0.5 & 0.5 & 0.8 \\
\hline & $\begin{array}{l}\text { Fiber strength reduc- } \\
\text { tion factor } f^{\prime}\end{array}$ & 0.3 & 0.3 & - \\
\hline
\end{tabular}

${ }^{a}$ After Li et al. (1995) and Kanda and Li (1999).

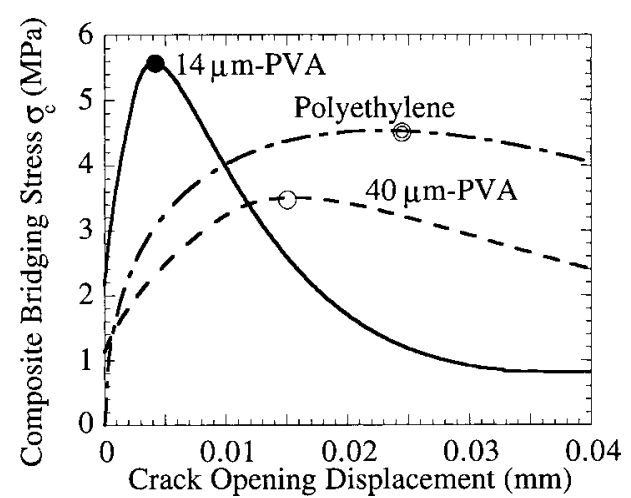

FIG. 6. Composite Bridging Performance in Theory

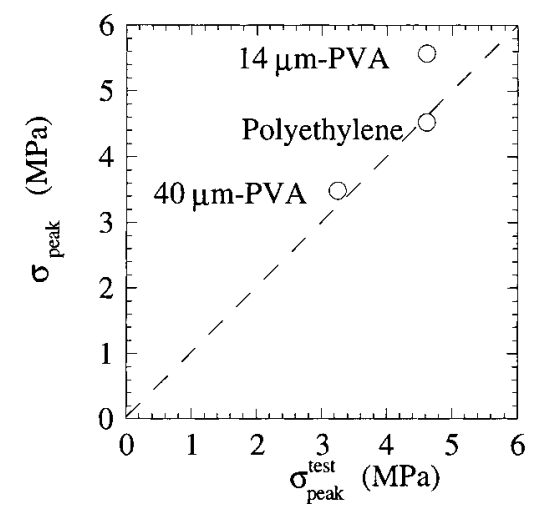

FIG. 7. Comparison of Peak Bridging Stress between Theory and Test

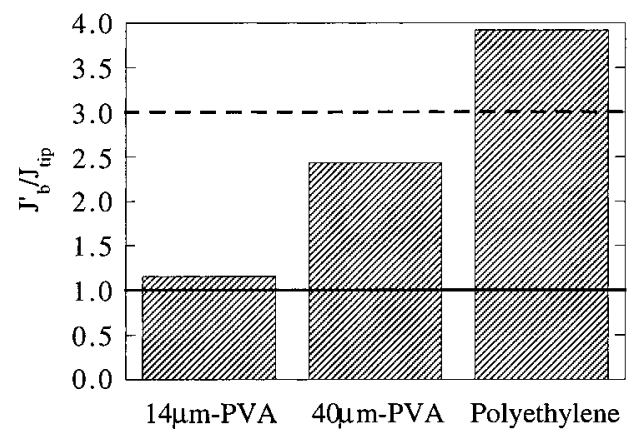

FIG. 8. Comparison of Energy Performance Estimation Result

estimated with matrix fracture toughness $K_{m}$ and matrix elastic modulus $E_{m}$ in Table $2\left(J_{\text {tip }}=K_{m}^{2} / E_{m}\right)$. The estimation results of $J_{b}^{\prime} / J_{\text {tip }}$ for the three composites are compared in Fig. 8, which demonstrates high $J_{b}^{\prime} / J_{\text {tip }}$ for the PE fiber composite, moderate for the 40- $\mu \mathrm{m}$-PVA fiber composite and low for the 14- $\mu \mathrm{m}$-PVA fiber composite. The solid line in Fig. 8 represents theoretical PSH condition with (6). This figure shows that $J_{b}^{\prime} / J_{\text {tip }} \geq 1$ is maintained for the three composites and implies that they should exhibit multiple cracking in theory. This estimation appears consistent with the tensile test results because these composites generate more than one crack. However, as pointed out earlier, these composites indicate very different degrees of multiple cracking, and only the PE composite showed saturated PSH behavior in practice. The more detailed investigation in the following section reveals the sources of this discrepancy in multiple cracking or PSH behavior as well as leading to more reliable verification for the proposed theory.

\section{Rationalizing Theory with PSH Intensity}

The PSH intensity has been examined recently using PE fiber composites by the authors (Kanda and Li 1998b). The 
research results indicated two types of PSH behavior: saturated and unsaturated. This classification is attributed to statistical variation of composite properties, which contributes to a gradual transition of multiple crack saturation. Satisfying $J_{b}^{\prime} / J_{\text {tip }} \geq$ 1 ensures that composites have sufficient potential for PSH behavior in the deterministic sense. However, for composites having $J_{b}^{\prime} / J_{\text {tip }}$ close to unity, statistical variation of micromechanical parameters (e.g., bond strength, fiber volume fraction, and/or matrix crack tip toughness) tends to lead to violating this condition thus causing an incomplete multiple cracking process. On the other hand, for composites with $J_{b}^{\prime} / J_{\text {tip }}$ much higher than unity, there is less chance to violate the PSH condition with sufficient potential margin in each occurrence of cracking. Therefore, much more cracks are likely developed in composites thus causing more intense PSH behavior. This intense multiple cracking process eventually is terminated when crack spacing becomes too close for transferring stress from fibers at a crack plane to create another matrix crack (Wu and Li 1995). This type of multiple cracking (saturated multiple cracking) leads to saturated PSH behavior.

The investigated composites can be classified into the foregoing two PSH categories. Both PVA fiber composites appear to show unsaturated PSH behavior in the experiment. This is likely caused by rather low $J_{b}^{\prime} / J_{\text {tip }}$ as demonstrated in Fig. 8, which causes high likelihood for violating PSH criteria $J_{b}^{\prime} / J_{\text {tip }} \geq 1$ in practice. For the PVA fiber composites, limited number of cracks consecutively developed may have been followed by violation of the $\sigma_{\text {peak }}>\sigma_{a}$ criteria on one of these crack planes, leading to immediate failure (displacement softening). On the other hand, the PE fiber composite appears to indicate saturated PSH behavior. This composite has enough potential for saturated PSH behavior, having $J_{b}^{\prime} / J_{\text {tip }}$ beyond 4 . Indeed, this composite was verified to show almost the same crack spacing as saturated crack space computed analytically (Wu and Li 1995).

Finally, the measure of PSH intensity $\varepsilon_{c u}$ was correlated with $J_{b}^{\prime} / J_{\text {tip }}$ in Fig. 9. In this figure, the range of $J_{b}^{\prime} / J_{\text {tip }}$ is divided into area I, II, and III, including the aforementioned two categories of PSH composites. In area I with an upper bound of $J_{b}^{\prime} / J_{\text {tip }}=1$, the PSH condition in (6) is violated so that PSH behavior is impossible. This represents the condition and behavior of conventional fiber-reinforced concrete. Areas II and III correspond to unsaturated and saturated PSH behavior, respectively. The $J_{b}^{\prime} / J_{\text {tip }}=3$ was suggested for the boundary for these two PSH behaviors using uniaxial tensile test data of PE composites with different volume fractions (Kanda and $\mathrm{Li}$ 1998b). The discussion in the foregoing paragraph suggests that the PVA fiber composites fall into area II with less $\varepsilon_{c u}$, but the PE composite is in area III with higher $\varepsilon_{c u}$. The test results in Fig. 9 confirm this expectation. This figure shows that the 14- $\mu$ m-PVA fiber composite is located at the boundary between area I and II with low $\varepsilon_{c u}$. Furthermore, the $40-\mu \mathrm{m}$ PVA fiber composite is in the middle of area II, and corre-

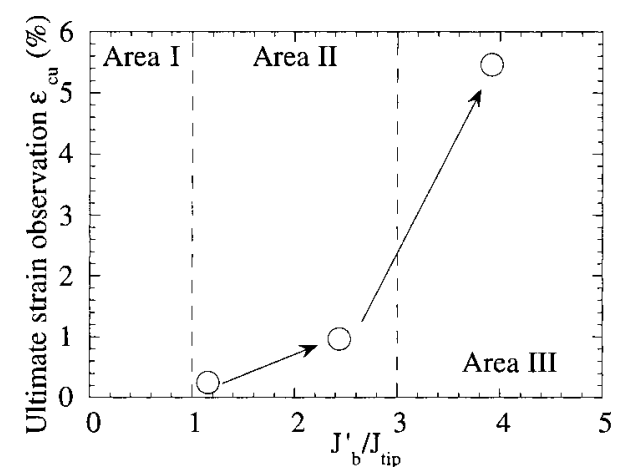

FIG. 9. Effect of Energy Margin on Composite Ultimate Strain sponding $\varepsilon_{c u}$ is higher than that of the 14- $\mu$ m-PVA fiber case. The third composite, the PE fiber system, is placed in area III with much higher $\varepsilon_{c u}$ than the other two, where saturated PSH behavior in theory is expected and is consistent with the test results. These observations appear to support the use of the current model for designing PSH composites that involve fiber rupture and chemical bond dominant fiber-matrix interface.

The comparison of the three $\sigma_{c}-\delta$ curves in Fig. 6 is very insightful from the viewpoint of the PSH composite design. To maintain high $J_{b}^{\prime}$ it is necessary to extend both $\sigma_{\text {peak }}$ and $\delta_{\text {peak }}$, which is achieved, e.g., by using the PE fiber in the composite. The PE fiber composite has moderate $\sigma_{\text {peak }}$ and very large $\delta_{\text {peak }}$ among the three composites. On the other hand, the 14- $\mu \mathrm{m}$-PVA fiber composite ensures high $\sigma_{\text {peak }}$ but possesses low $\delta_{\text {peak }}$, thus failing in maintaining high $J_{b}^{\prime}$. This discrepancy in performance is attributed to the specific combination of micromechanics parameters in these composites. To effectively design PSH composites, it is necessary to clarify the effects of these parameters on composite performance. This clarification is achieved via parametric study in the next section.

\section{PARAMETRIC STUDY}

\section{Scheme of Parametric Study}

A parametric study is conducted to reveal the effects of micromechanical parameters on composite performance, thus yielding useful information for composite optimization. Composite performance may be represented by peak bridging stress $\bar{\sigma}_{\text {peak }}$ and complementary energy $\bar{J}_{b}^{\prime}$. This is because $\bar{\sigma}_{\text {peak }}$ dominates load-bearing capacity of composites. Furthermore, $\bar{J}_{b}^{\prime}$ controls deformation capacity (ductility) of composites having identical matrix mix proportion (identical matrix crack tip toughness $J_{\text {tip }}$ ). Load-bearing capacity and ductility are critical performances for structural materials. These two properties are governed by the same primary micromechanical parameters, $E_{f} / \lambda, \sigma_{d s} / \lambda, \sigma_{f u}^{n} / \lambda, f$, and $f^{\prime}$, as for the $\bar{\sigma}_{c}-\bar{\delta}$ relation [see (1)].

This parametric study deploys a reference composite system whose micromechanical primary parameters are $E_{f} / \lambda=50, \sigma_{d s} l$ $\lambda=0.125, \sigma_{f u}^{n} / \lambda=2, f=0.5$, and $f^{\prime}=0.3$. These micromechanical parameters were determined as the intermediate properties among those of the three composite systems experimentally investigated in this study as summarized in Table 2 . The first three primary parameters $E_{f} / \lambda, \sigma_{d s} / \lambda$, and $\sigma_{f u}^{n} / \lambda$ are distributed broadly; i.e., $16<E_{f} / \lambda<250,0.45<$ $\sigma_{f u}^{n} / \lambda<5.1$, and $0<\sigma_{d s} / \lambda<0.42$ as shown in Table 3. It should be noted that the upper bound of $\sigma_{d s} / \lambda$ was calculated by assuming the PE fiber has chemical bond strength the same as that of the 14- $\mu \mathrm{m}$-PVA fiber (33.6 MPa). This assumption is likely realistic because interface properties can be tailored via various operations, such as deploying coupling agent and plasma treatment. Specifically, it is possible to modify surface chemistry of the PE fiber from hydrophobic to hydrophilic nature with plasma treatment (Li et al. 1996). Such modification results in high chemical bond strength in the PE fiber.

The parametric study was conducted by varying one of the five primary parameters within realistic system. The first three primary parameters were changed within the range in Table 3.

TABLE 3. Magnitude of Primary Parameters for Composites

\begin{tabular}{c|c|c|c}
\hline \hline $\begin{array}{c}\text { Primary } \\
\text { parameter } \\
(1)\end{array}$ & $\begin{array}{c}40-\mu m-P V A \\
\text { composite } \\
(2)\end{array}$ & $\begin{array}{c}14-\mu m-P V A \\
\text { composite } \\
(3)\end{array}$ & $\begin{array}{c}\text { PE } \\
\text { composite } \\
(4)\end{array}$ \\
\hline$E_{f} / \lambda$ & 16 & 16 & 250 \\
$\sigma_{f u}^{n} / \lambda$ & 0.61 & 0.45 & 5.1 \\
$\sigma_{d s} / \lambda$ & 0.057 & 0.038 & 0 \\
& & & $(0.42)^{\mathrm{a}}$ \\
\hline
\end{tabular}

${ }^{\mathrm{a}}$ Assuming chemical bond strength $\tau_{s}=33.6 \mathrm{MPa}$. 
The $f$ and $f^{\prime}$ were increased up to reported maximum values (Kanda and Li 1998a; Li et al. 1990).

\section{Result of Parametric Study}

First, the influence of the chemical bond is examined in Fig. 10 , which shows the effects of $\sigma_{d s} / \lambda$ on $\bar{\sigma}_{\text {peak }}$ and $\bar{J}_{b}^{\prime}$. In this figure, $\bar{\sigma}_{\text {peak }}$ slightly increases (approximately $10 \%$ ) but $\bar{J}_{b}^{\prime}$ significantly decreases to less than half as $\sigma_{d s} / \lambda$ increases from 0 to 0.3 . This descent in $\bar{J}_{b}^{\prime}$ is attributed to greatly reduced $\bar{\delta}_{\text {peak }}$ and increased $\bar{\sigma}_{c}(\bar{\delta}=0)$. Reduced $\bar{\delta}_{\text {peak }}$ is accompanied by higher stiffness of fiber bridging caused by stronger frictional and chemical bonding. Stronger chemical bonding also causes increased $\bar{\sigma}_{c}(\bar{\delta}=0)$. These trends are implied with $\sigma_{c^{-}}$ $\delta$ relations in Fig. 6. Fig. 6 indicates lower $\delta_{\text {peak }}$ of the $14-\mu \mathrm{m}-$ PVA and 40- $\mu$ m-PVA fiber composites, which have very high $\tau_{i}$ and $\tau_{s}$ as shown in Table 2, than those of the PE composite. Furthermore, Fig. 6 shows $\bar{\sigma}_{c}(\bar{\delta}=0)>0$ for these PVA composites but $\bar{\sigma}_{c}(\bar{\delta}=0)=0$ for the PE composite with $\tau_{s}=0$. This nonzero $\bar{\sigma}(\bar{\delta}=0)$ for the PVA composites is a result of neglecting elastic deformation of matrix foundation in modeling single fiber pullout behavior. The details of this assumption should be referred to in literature (Kanda and Li 1999). Consequently, the results in Fig. 10 reveal that the overall effect of chemical bond on composite performance is rather negative.

This finding for the effects of chemical bond is very important. This is because this finding clarifies the importance of limiting the chemical bond strength for high composite performance. Therefore, optimizing composites requires careful tailoring of fiber-matrix interface properties. Indeed, similar effects of chemical bonds have been reported for the performance of aligned short fiber-reinforced composites by Leung (1996). However, the current study eliminates the restriction in aligned fiber in the preceding study and physically clarified the sources for the negative effects of chemical bond.

The effects of nominal fiber strength $\sigma_{f u}^{n}$ are illustrated in Fig. 11, which shows ascending trend both in $\bar{\sigma}_{\text {peak }}$ and $\bar{J}_{b}^{\prime}$ with increase of $\sigma_{f u}^{n} / \lambda$. In this figure, $\bar{\sigma}_{\text {peak }}$ increases almost proportionally to $\sigma_{f u}^{n} / \lambda$. Similar to this result, an approximation formula for predicting peak bridging stress $\sigma_{\text {peak }}$, which has been proposed for a composite with fiber rupture in a previous study (Maalej et al. 1995), assumed that $\sigma_{\text {peak }}$ is proportional to $\sigma_{f u}^{n}$. However, the influence on $\bar{J}_{b}^{\prime}$ is much more remarkable; doubling of $\sigma_{f u}^{n} / \lambda$ (from 1 to 2 ) results in almost an order of magnitude higher in $\bar{J}_{b}^{\prime}$. This result shows that the magnitude of $\sigma_{f u}^{n}$ remarkably affects the performance of fiber rupturetype composites especially in terms of strain capacity. Therefore, for prediction purposes, $\sigma_{f u}^{n}$ should be determined precisely according to the actual condition of fibers in composites because apparent fiber strength in composites was reported lower than that obtained by standard fiber strength tests even

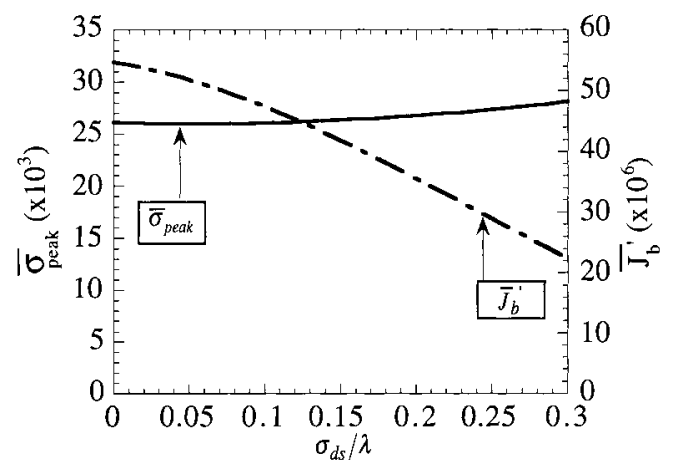

FIG. 10. Effect of Chemical Bond Strength on Peak Bridging Stress and Complementary Energy $\left(E_{f} / \lambda=50, \sigma_{f u}^{n} / \lambda=2, f=0.5\right.$, and $f^{\prime}=0.3$ ) without inclining angle (Kanda and Li 1998a). Furthermore, for actual material design, high-strength fiber is indispensable to establish high-performance composites.

Fig. 12 illustrates the negative effects of snubbing coefficient $f$. Higher $f$ has been reported to improve remarkably composite performance involving no fiber rupture in terms of stress and strain capacities [e.g., Li and Leung (1992)]. However, Fig. 12 indicates that higher $f$ can significantly reduce $\bar{J}_{b}^{\prime}$ of a composite. In this figure, the range of $0.5<f<1.0$ appears realistic for actual fiber composites because $f=1.0,0.7$, and 0.5 were, respectively, reported for a nylon fiber, a polypropylene fiber (Li et al. 1990), and a PE fiber (Li et al. 1995). The $\bar{J}_{b}^{\prime}$ declines by approximately $70 \%$ when $f$ increases from 0.5 to 1.0 whereas $\bar{\sigma}_{\text {peak }}$ is stable in this range.

The negative effects of apparent strength-reduction factor $f$ are illustrated in Fig. 13, which shows both $\bar{\sigma}_{\text {peak }}$ and $\bar{J}_{b}^{\prime}$ decrease with increase of $f^{\prime}$. This figure indicates a stronger effect of $f$ on $\bar{J}_{b}^{\prime}$ than on $\bar{\sigma}_{\text {peak }}$. Increase of $f^{\prime}$ from 0 to 0.3

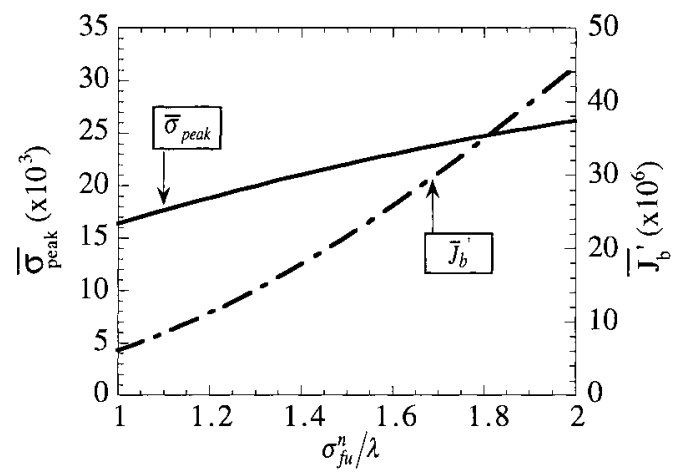

FIG. 11. Effect of Nominal Fiber Strength on Peak Bridging Stress and Complementary Energy $\left(E_{f} / \lambda=50, \sigma_{d s} / \lambda=0.125, f=\right.$ 0.5 , and $f^{\prime}=0.3$ )

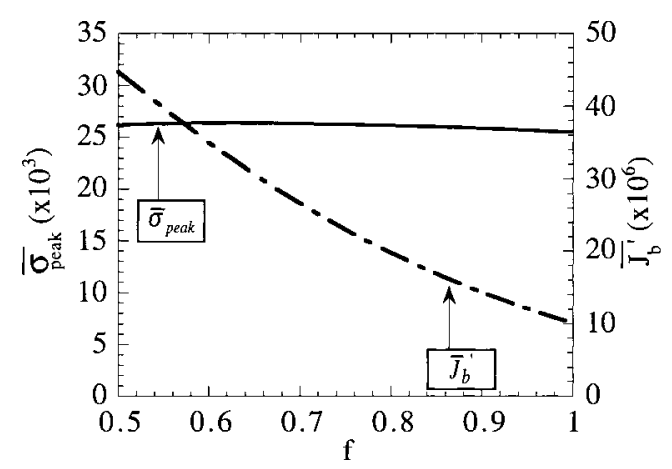

FIG. 12. Effect of Snubbing Coefficient on Peak Bridging Stress and Complementary Energy $\left(E_{f} / \lambda=50, \sigma_{d s} / \lambda=0.125\right.$, $\sigma_{f u}^{n} / \lambda=2$, and $f^{\prime}=0.3$ )

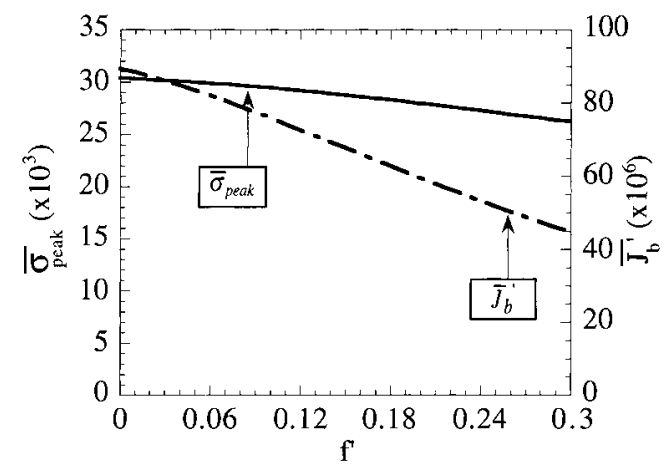

FIG. 13. Effect of Fiber Strength-Reduction Factor on Peak Bridging Stress and Complementary Energy $\left(E_{f} / \lambda=50, \sigma_{d s} / \lambda=\right.$ $0.125, \sigma_{f u}^{n} / \lambda=2$ and $f=0.5$ ) 


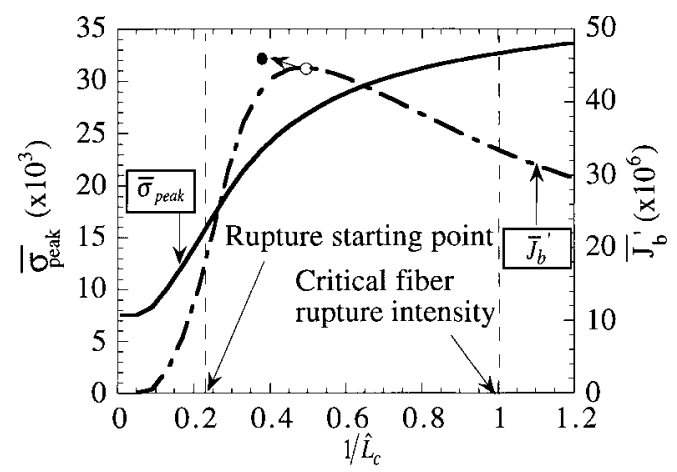

FIG. 14. Effect of Fiber Rupture Intensity on Peak Bridging Stress and Complementary Energy $\left(E_{f} / \sigma_{f u}^{n}=25, \sigma_{d s} / \sigma_{f u}^{n}=0.0625\right.$, $f=0.5$, and $f^{\prime}=0.3$ )

reduces $\bar{\sigma}_{\text {peak }}$ by $20 \%$ and $\bar{J}_{b}^{\prime}$ by $50 \%$. For accuracy of predicting composite performance, this result suggests that neglecting $f^{\prime}$ causes larger error in prediction of $\bar{J}_{b}^{\prime}$ than in $\bar{\sigma}_{\text {peak }}$.

Finally, the effect of fiber rupture intensity is studied. The fiber rupture intensity, represented by the reciprocal of $\bar{L}_{c}$ (Appendix I), is a measure of the longest embedment length of a fiber that pulls out without rupture. Therefore, longer $\hat{L}_{c}$ implies a smaller population of rupture fiber. For a straight aligned fiber with friction bond, $\bar{J}_{b}^{\prime}$ will show a maximum at $1 / \hat{L}_{c}=1$ (critical fiber rupture intensity, hereafter), where fiber rupture initiates. More fiber rupture is expected for longer fibers, which means $1 / \hat{L}_{c}>1$, thus diminishing the complementary fiber bridging energy. In the present study, composites with inclined fibers whose apparent strength is sensitive to inclining angle are considered. Further, the contribution of the chemical bond leads to increasing the amount of fiber rupture. The computed $\bar{J}_{b}^{\prime}$ therefore is expected to peak at a lower rupture intensity. This is found to be the case, as shown in Fig. 14, with peak occurring at $1 / \hat{L}_{c} \approx 0.5$ for the composite system with $E_{f} / \sigma_{f u}^{n}=25, \sigma_{d s} / \sigma_{f u}^{n}=0.0625, f=0.5$, and $f^{\prime}=$ 0.3 . This optimal rupture intensity is located between critical rupture intensity and rupture starting point, which is obtained by equating $L_{f}$ to $L_{r}$ and represents $1 / \hat{L}_{c}$ below which no fiber rupture can occur at any angle $\phi$. These two limits are indicated in Fig. 14, which also shows that the peak bridging stress rises monotonically with rupture intensity.

It should be noted that the reference composite system used for Figs. $10-13$ is identical to the system at $1 / \hat{L}_{c} \approx 0.5$ in Fig. 14. Indeed, the reference system in the parametric study has been selected so as to have optimal value of $1 / \hat{L}_{c}$ and to have $1 / \hat{L}_{c}$ located between the foregoing two limits even with the variation of the fundamental parameters as in Figs. 10-13 while the optimal rupture intensity and corresponding $\bar{J}_{b}^{\prime}$ vary with the magnitudes of these parameters. For example, increasing $f$ from 0.5 to 1.0 yields lower optimal rupture intensity and slightly higher corresponding $\bar{J}_{b}^{\prime}$. This optimal point for $f$ $=1.0$ is indicated with " $\bullet$ ' in Fig. 14 .

\section{DESIGN IMPLICATION}

The parametric study in the previous section reveals the effects of fundamental micromechanical parameters and suggests the potential of the proposed theory as a powerful composite design tool. This possibility is further pursued by analyzing the PVA fiber composites with the current theory. The PVA fiber composites have been clarified to have insufficient energy performance for saturated PSH behavior (Fig. 9). The foregoing parametric study indicates that energy performance can be optimized by controlling fiber rupture intensity, which is expressed with $1 / \hat{L}_{c}$. Therefore, estimating rupture intensity for these two composites may facilitate understanding the

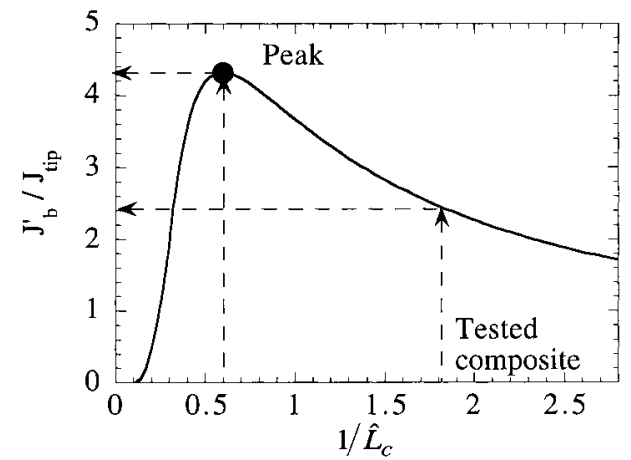

FIG. 15. Fiber Rupture Intensity of 40- $\mu \mathrm{m}$-PVA Fiber Composite and Its Optimization For Energy Performance

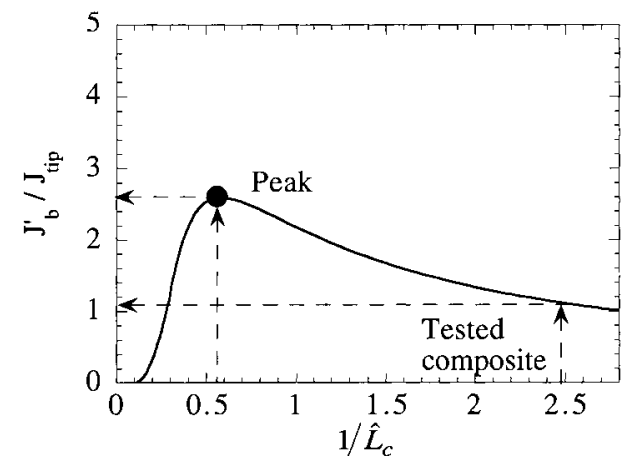

FIG. 16. Fiber Rupture Intensity of 14- $\mu \mathrm{m}$-PVA Fiber Composite and Its Optimization for Energy Performance

sources for their insufficient energy performance. This estimation result is depicted in Figs. 15 and 16, in which the effects of $1 / \hat{L}_{c}$ on $J_{b}^{\prime} / J_{\text {tip }}$ are illustrated. Fig. 15 indicates that the maximum $J_{b}^{\prime} / J_{\text {tip }}(=4.5)$ is at $1 / \hat{L}_{c}=0.6$ whereas the 40 $\mu \mathrm{m}$-PVA fiber composite has $J_{b}^{\prime} / J_{\text {tip }}=2.4$ at $1 / \hat{L}_{c}=1.8$. This shows that this composite has too intense fiber rupture, which deteriorates $J_{b}^{\prime} / J_{\text {tip }}$ to $56 \%$ of the maximum. Hence if optimization is achieved for this composite, its energy performance can be enhanced to even higher than that of the PE fiber composite shown in Fig. 8, which is located in area III in Fig. 9 and leads to saturated PSH behavior. Next, Fig. 16 shows that the tested 14- $\mu \mathrm{m}$-PVA fiber composite is far from the optimal state. This composite's $1 / \hat{L}_{c}$ reaches near 2.5 while the maximum $J_{b}^{\prime} / J_{\text {tip }}=2.6$ is attained at $1 / \hat{L}_{c}=0.55$. This discrepancy attributes to an actual $J_{b}^{\prime} / J_{\text {tip }}$ less than $50 \%$ of that of the optimum. The optimized 14- $\mu \mathrm{m}-\mathrm{PVA}$ fiber system is expected to show better PSH behavior but still inadequate saturated PSH behavior having $J_{b}^{\prime} / J_{\text {tip }}<3$.

The foregoing optimization relating PVA fiber's rupture intensity can be achieved by reducing chemical or frictional bond strength of the fiber-matrix interface. This may be realized by modifying the chemical characteristics of the fiber surface. For example, the plasma treatment described earlier could be appropriate for this purpose ( $\mathrm{Li}$ et al. 1996). This treatment has been conducted in atmospheres such as with Argon and enhances bond strength of the fiber-matrix interface by adding hydroxyl groups to the fiber surface. For PVA fiber the opposite effects may be achieved using a different selection of atmosphere like fluorocarbon, which is expected to restrict hydroxyl groups on the surface. It should be noted that reducing PVA fiber length does not contribute to the optimization. Fiber length reduction causes reduction of the absolute value of $J_{b}^{\prime}$ while diminishing the fiber rupture intensity.

\section{CONCLUSIONS}

This study is aimed at developing a comprehensive design theory to achieve PSH behavior in composites containing 
high-performance hydrophilic fibers such as PVA fibers. The new design theory combines the energy approach for the PSH condition and a new bridging law, which accounts for chemical bond and fiber rupture.

The design condition for PSH behavior was first derived in terms of micromechanical parameters. This design theory was validated deploying the uniaxial tensile tests of two PVA fiber composites with different PSH intensity. Together with the tensile test results of the PE fiber composite showing saturated PSH behavior, the proposed theory was found to evaluate reasonably tensile strain behavior of PSH composites via the toughness ratio $J_{b}^{\prime} / J_{\text {tip. }}$.

The mechanical performance of the hydrophilic fiber composites was examined through parametric studies by using the proposed theory. Focus was placed on the composite peak bridging stress $\sigma_{\text {peak }}$ and complimentary energy $J_{b}^{\prime}$, which govern the ultimate strength and strain capacity of composites, respectively. The investigation revealed the following: (1) Increase of the fiber rupture intensity (measured by $1 / \hat{L}_{c}$ ) enhances $\sigma_{\text {peak }}$, but an optimum rupture intensity exists for maximum $J_{b}^{\prime}$ of the composites; and (2) the fundamental parameters $\sigma_{d s}, f$, and $f^{\prime}$ significantly reduce $J_{b}^{\prime}$ for composites with $1 / \hat{L}_{c}$ close to the optimum.

Based on the results of the parametric study, the two PVA fiber composites were examined further to achieve better PSH behavior. As a result, these composites were found to have too intense of a fiber rupture, which deteriorates $J_{b}^{\prime} / J_{\text {tip }}$ and leads to lower strain capacity. Reducing this rupture intensity was proposed to achieve better strain capacity, which may be achieved by restricting chemical/frictional bond strength.

The proposed design approach can expand the variety of fiber types, which can be utilized for PSH-RSFRCCs. As a result, PSH-RSFRCCs may increase their practicalities, such as more reasonable cost and easy processing. To satisfactorily achieve this goal, further investigation is needed for rupture type PSH-RSFRCCs. Actual design practice for these composites should be accumulated using various types of fibers. An example of such practices will be reported in a subsequent study.

\section{APPENDIX I. FULL EXPRESSION OF BRIDGING LAW}

For $\hat{L}_{r}<2$ and $1<\hat{L}_{c}$

$$
\sigma_{c}=\left\{\begin{array}{c}
\sigma_{0 i d} C_{A} G\left(\frac{\pi}{2}, f\right) \text { for } 0 \leq \frac{\delta}{\delta_{u}\left(\phi=\frac{\tau}{2}\right)} \leq 1 \\
\sigma_{0 i}\left\{\begin{array}{l}
{ }_{d} C_{A} G\left(\phi_{c}, f\right)+{ }_{d} C_{B 2} A\left(\phi_{c},-f-2 f^{\prime}\right) \\
+{ }_{d} C_{B 3} A\left(\phi_{c},-f^{\prime}\right)+{ }_{d} C_{B 4} A\left(\phi_{c}, f\right)
\end{array}\right\} \\
\text { for } 1<\frac{\delta}{\delta_{u}\left(\phi=\frac{\pi}{2}\right)}
\end{array}\right.
$$

For $\hat{L}_{c}<1$

$$
\sigma_{c}=\left\{\begin{array}{c}
\sigma_{0 i d} C_{A} G\left(\frac{\pi}{2}, f\right) \text { for } 0 \leq \frac{\delta}{\delta_{u}\left(\phi=\frac{\pi}{2}\right)} \leq 1 \\
\sigma_{0 i}\left\{\begin{array}{l}
{ }_{d} C_{A} G\left(\phi_{c}, f\right)+{ }_{d} C_{B 2} A\left(\phi_{c},-f-2 f^{\prime}\right) \\
+{ }_{d} C_{B 3} A\left(\phi_{c},-f^{\prime}\right)+{ }_{d} C_{B 4} A\left(\phi_{c}, f\right)
\end{array}\right\} \\
\text { for } 1<\frac{\delta}{\delta_{u}\left(\phi=\frac{\pi}{2}\right)}
\end{array}\right.
$$

where

$$
\begin{aligned}
& \sigma_{0 i}=\frac{V_{f} \tau_{i}}{2}\left(\frac{L_{f}}{d_{f}}\right) \\
& { }_{d} C_{A}=-\gamma+2 \gamma^{1 / 2}+\left(\frac{\sigma_{d s}}{\lambda}\right)^{2}, \quad{ }_{d} C_{B 2}=\left(\frac{\sigma_{f u}^{n}}{\lambda}\right)^{2}, \\
& { }_{d} C_{B 3}=-2 \xi\left(\frac{\sigma_{f u}^{n}}{\lambda}\right)^{2}, \quad{ }_{d} C_{B 4}=\left[\xi\left(\frac{\sigma_{f u}^{n}}{\lambda}\right)\right]^{2}, \quad{ }_{d} C_{C}=1 \\
& \gamma=\left(\frac{E_{f}}{\lambda}\right) \bar{\delta}+\left(\frac{\sigma_{d s}}{\lambda}\right)^{2}, \quad \xi=\left(\frac{\sigma_{d s}}{\lambda}\right) /\left(\frac{\sigma_{f u}^{n}}{\lambda}\right) \\
& \int \frac{\pi}{2} \text { for } 0 \leq \frac{\delta}{\delta_{u}\left(\phi=\frac{\pi}{2}\right)} \leq 1 \\
& \phi_{c}(\hat{\delta})=\left\{-\frac{1}{2\left(f+f^{\prime}\right)} \ln \left[\left(\frac{E_{f}}{\lambda}\right) \bar{\delta} /\left(\frac{\sigma_{f u}^{n}}{\lambda}\right)^{2}+\xi^{2}\right]\right. \\
& \text { for } 1<\frac{\delta}{\delta_{u}\left(\phi=\frac{\pi}{2}\right)} \\
& \frac{\delta}{\delta_{u}\left(\phi=\frac{\pi}{2}\right)}=\frac{\left(\frac{E_{f}}{\lambda}\right) \bar{\delta}}{\left(\frac{\sigma_{d s}}{\lambda}\right)^{2}\left[e^{-\left(f+f^{\prime}\right) \pi}-\xi^{2}\right]} \\
& \hat{L}_{c}=\left(\frac{\sigma_{f u}^{n}}{\lambda}\right)(1-\xi), \quad \hat{L}_{r}=2\left(\frac{\sigma_{f u}^{n}}{\lambda}\right)\left[e^{-\left(f+f^{\prime}\right) \pi / 2}-\xi\right] \\
& G(\phi, \alpha)=\frac{1}{\alpha^{2}+4}\left\{e^{\alpha \phi}[\alpha \sin 2 \phi-2 \cos 2 \phi]+2\right\} \\
& A(\phi, \alpha)=\frac{1}{\alpha^{2}+4}\left\{e^{\alpha \phi}[2 \cos 2 \phi-\alpha \sin 2 \phi]+2 e^{(\pi / 2) \alpha}\right\} \\
& B\left(\phi_{1}, \phi_{2}, \alpha\right)=\frac{1}{\alpha^{2}+4}\left\{e^{\alpha \phi_{2}}\left[\alpha \sin 2 \phi_{2}-2 \cos 2 \phi_{2}\right]\right. \\
& \left.+e^{\alpha \phi_{1}}\left[2 \cos 2 \phi_{1}-\alpha \sin 2 \phi_{1}\right]\right\}
\end{aligned}
$$

\section{ACKNOWLEDGMENTS}

This paper was completed when V.C. Li was a visiting professor at the University of Tokyo, Japan. Part of this research was supported by Grant CMS-EQ-9601262 from the National Science Foundation to the University of Michigan at Ann Arbor. T. Kanda was supported by Kajima Corporation (Tokyo). The writers thank Mr. J. Hikasa in Kuraray Co., Ltd (Osaka, Japan) for helpful discussion and material supply. T. Kanda acknowledges valuable advice from Dr. H. C. Wu, Dr. Z. Lin, and Dr. M. Maalej.

\section{APPENDIX II. REFERENCES}

Akihama, S., Suenaga, T., Nakagawa, H., and Suzuki, K. (1985). "Experimental study of vinylon fiber reinforced cement composites "VFRC" (Part I)." Kajima Annu. Tech. Rep., Kajima Corporation, 33, 123-128 (in Japanese).

Aveston, J., Cooper, G. A., and Kelly, A. (1971). "Single and multiple fracture." The properties of fiber composites, Guildford, U.K., 15-26.

Betterman, L. R., Ouyang, C., and Shah, S. P. (1995). "Fiber-matrix interaction in microfiber-reinforced mortar." Adv. Cement Based Mat., 3(2), 53-61.

Evans, A. G., Domergue, J. M., and Vagaggini, E. (1994). "Methodology for relating the tensile constitutive behavior of ceramic matrix composites to constituent properties." J. Am. Ceramic Soc., 77(6), 14251435.

Garcia, S., Naaman, A. E., and Pera, J. (1997). "Experimental investigation on the potential use of poly(vinyl alcohol) short fibers in fiber- 
reinforced cement-based composites.' Mat. and Struct., Paris, 30(Jan.Feb.), 43-52.

Kanda, T., and Li, V. C. (1999). "Effect of apparent strength and fibermatrix interface properties on crack bridging in cementitious composites." J. Engrg. Mech., ASCE, 125(3).

Kanda, T., and Li, V. C. (1998a). "Interface property and apparent strength of a high strength hydrophilic fiber in cement matrix.' J. Mat. in Civ. Engrg., ASCE, 10(1), 5-13.

Kanda, T., and Li, V. C. (1998b). "Multiple cracking sequence and saturation in fiber reinforced cementitious composites." Concrete Res. and Technol., Tokyo, 9(2), 1-15.

Leung, C. K. Y. (1996). "Design criteria for pseudoductile fiber-reinforced composites." J. Engrg. Mech., ASCE, 122(1), 10-14.

Li, V. C. (1993). "From micromechanics to structural engineering - the design of cementitious composites for civil engineering applications." J. Struct. Mech. Earthquake Engrg., Tokyo, 10(2), 37-48.

Li, V. C., and Leung, K. Y. (1992). "Steady-state and multiple cracking of short random fiber composites." J. Engrg. Mech., ASCE, 118(11), $2246-2263$.

Li, V. C., Mishra, D. K., and Wu, H. C. (1995). "Matrix design for pseudo-strain hardening fiber reinforced cementitious composites." Mat. and Struct., Paris, 28, 586-595.

Li, V. C., Wang, Y., and Backer, S. (1990). "Effect of inclining angle, bundling, and surface treatment on synthetic fiber pull-out from a cement matrix." Composites, 21(2), 132-140.

Li, V. C., Wu, H. C., and Chan, Y. W. (1996). "Effect of plasma treatment of polyethylene fibers on interface and cementitious composite properties." J. Am. Ceramics Soc., 79(3), 700-704.

Maalej, M., Li, V. C., and Hashida, T. (1995). "Effect of fiber rupture on tensile properties of short fiber composites." J. Engrg. Mech., ASCE, 121(8), 903-913.

Marshall, D. B., and Cox, B. N. (1988). “A J-integral method for calculating steady-state matrix cracking stresses in composites." Mech. of Mat., 7, 127-133.

Marshall, D. B., Cox, B. N., and Evans, A. G. (1985). "The mechanics of matrix cracking in brittle-matrix fiber composites." Acta Metallurgica, 33(11), 2013-2021.
Naaman, A. E., and Reinhardt, H. W. (1995). High performance fiber reinforced cement composites. E \& FN Spon, London.

Spearing, S. M., and Zok, F. W. (1993). "Stochastic aspects of matrix cracking in brittle matrix composites." J. Engrg. Mat. and Technol., $115,314-318$.

Wu, H. C., and Li, V. C. (1995). "Stochastic process of multiple cracking in discontinuous random fiber reinforced brittle matrix composites." Int. J. of Damage Mech., 4(1), 83-102.

\section{APPENDIX III. NOTATION}

The following symbols are used in this paper:

$d_{f}=$ fiber diameter;

$E_{c}=$ elastic modulus of composite;

$E_{f}=$ elastic modulus of fiber;

$E_{m}=$ elastic modulus of matrix;

$f=$ snubbing coefficient;

$f^{\prime}=$ fiber strength-reduction factor;

$J_{\text {tip }}=$ crack tip toughness;

$J_{b}^{\prime}=$ complementary energy of $\sigma_{c}-\delta$ curve;

$L_{c}=$ maximum critical fiber embedment length;

$L_{f}=$ fiber length;

$L_{r}=$ minimum fiber length for fiber rupture;

$V_{f}=$ volume fraction of fiber;

$\delta=$ crack opening displacement;

$\delta_{\text {peak }}=$ crack opening displacement at $\sigma_{\text {peak }}$;

$\sigma_{c}=$ crack bridging stress of composite;

$\sigma_{\text {peak }}=$ peak bridging stress of composite;

$\sigma_{f u}^{n}=$ nominal tensile strength of fiber;

$\tau_{i}=$ frictional bond strength;

$\tau_{s}=$ chemical bond strength; and

$\phi=$ fiber inclining angle. 